\title{
QoS Constraints_Based Multicast Routing Algorithm Using CACO
}

\author{
Yang $\mathrm{Hao}^{1}$, Xian Zhengzheng ${ }^{2}$, Yang Lei $^{1}$ \\ 1. College of Computer Science and Engineering, University of Electronic Science and Technology of China, Chengdu, \\ Sichuan, China \\ 2. College of Computer Science and Technology, Guang Dong University of Finance, Guangzhou, Guangdong, china \\ muyibaigao@uestc.edu.cn
}

\begin{abstract}
QoS (Quality of Service) constraints_based multicast routing is one of major issues in distributed multimedia on the network. An improved algorithm based CACO (Chaos Ant Colony Optimization) is proposed in this paper for solving some routing problems such as the convergence speed, packet loss rate and bandwidth, etc. The algorithm utilizes the pheromone positive feedback effect to guide chaos search. The advantage of this scheme is that chaos initialization is adopted to improve individual quality and chaos perturbation is utilized to avoid the search being trapped in local optimum, meanwhile improve the packet delivery ratio and reduce the end-to-end delay. The experimental results show that the efficiency of the algorithm.
\end{abstract}

Keywords- QoS, QoS constraints, multicast routing, ACO, CACO, MANET

\section{INTRODUCTION}

Routing algorithm is a pivotal technology that underpins the network transmission, will develop with the appearance of the new network. Mobile Ad Hoc NETwork (MANET) is a new kind of wireless networks that it can self-organize and self-configureed. In this kind of networks, it doesn't require fixed infrastructure, the network topology constantly changes and all the mobile nodes are equal that could be a routing node transmit messages. Therefore, design a new dynamic routing protocol is important for ad hoc network[1].

The main problem to be solved by QoS routing algorithm is the constraints based multicast routing Path problem. Algorithms to solve this family of problems are known to be heuristics which can reduce the complexity of the path computation, however, at the expense of not attaining the optimal solution for the problem and finding just a feasible solution. The path computation algorithm is at the core of QoS routing strategies. Instead of using a shortest path algorithm based on statically configured metrics, as in traditional routing protocols, the algorithm must select several alternative paths that are able to satisfy a set of constraints regarding, for instance, end-to-end delay bounds and bandwidth requirements. However, the algorithms to solve such a problem have been shown to have, in general, high computational complexity[2] .

Therefor, there is a need to develop a unified routing protocol, which also results in overall network management in addition to just packet routing. In this attempt, this project looks at the characteristics of emergence behavior exhibited in natural environments by studying the emergence behavior in ants and presents a unified solution for network management through routing.
The remainder of this paper is organized as follows. In Section 2, we present the QoS constraints_based multicast routing method, especially, emphasize on the chaos ant pheromone model. In Section 3, the performance of the proposed algorithm is analyzed through Experiment. Finally, a conclusion is given in Section 4.

\section{QOS CONSTRAINTS_BASED MULTICAST ROUTING METHOD}

\section{A. MANET QoS Model}

Mobile Ad hoc NETwork (MANET) is a self-organized and self-managed network. Effective routing algorithm is one of its key technologies due to its dynamic network topology[3]. Let $G=(V, E)$ stand for the network model. $V$ is the network nodes and $E$ is the network links. $T=(V 1, E 1) \in G$ is the current multicast tree. $s t \in(V-V 1)$ is destination nodes to join the multicast group. $p=p(s c, d t)$ is a selected path from the source node $s c$ to the destination $d t, e$ is a link on the path of $p$, namely $e \in p$. Each link of the trees $T$ corresponds to the four positive real numbers $(\mathrm{De}, \mathrm{DJ}, \mathrm{BWe}, \mathrm{Ce})$. Among them, $D e, D J e, B W e$ and $C e$ denote respectively the transmission delay, transmission delay jitter, bandwidth available and costs of $e$. QoS parameters in common used are showed in math as follows[4]

$$
\begin{aligned}
& \quad \operatorname{delay}(p)=\sum_{e \in p} \operatorname{delay}(e) \\
& \text { Delay: } \\
& \text { delay }(e) \text { is the delay of chain } e ; \\
& \quad \text { delay }-\operatorname{jitter}(p)=\sum_{e \in P} \operatorname{dj}(e) \\
& \text { Delay jitter: } \\
& \text { dj }(e) \text { is the delay jitter of chain } e \text {; } \\
& \text { Bandwidth: bandwidth }(p)=\min \{b(e)\} \\
& b(e) \text { is the bandwidth of chain } e \text {; } \\
& \qquad \operatorname{cost}(p)=\sum_{e \in p} \operatorname{cost}(e) \\
& \text { Cost: } \operatorname{cost}(e) \text { is the cost of chain } e \text {; }
\end{aligned}
$$

For the node $\mathrm{d}$ to join the tree, QoS Constraints_Based Multicast Routing is to find a path $p(s, d)$ to meet the following conditions:

Delay constraints, namely: 


$$
\operatorname{delay}(p) \leq D
$$

Delay jitter constraints, namely:

$$
\text { delay-jitter }(p) \leq D J
$$

Bandwidth constraints, namely:

$$
\operatorname{bandwidth}(p) \geq B
$$

$$
\min \{\operatorname{cost}(p)\}
$$

$D, D J, B$ indicate respectively the multicast tree's delay constraints, delay jitter constraints, bandwidth constraints. $S$ is the random node of the tree.

\section{B. MANET QoS Routing Using ACO}

$\mathrm{ACO}$ (Ant Colony Optimization) is a heuristic approach for solving hard combinatorial optimization problems. It was first proposed by M.Dorigo, and had been successfully used to solve Traveling Salesman Problem. Since then, ACO was applied to network's routing by Gianni Di Caro and Marco Dorigo, called AntNet. Agent is mobile code which has a little intelligent, can move itself from node to node, cooperating with others to perform complex tasks in a distributed manner. These agents have obvious advantages such as reducing network overload greatly, running independent and asynchronous, adapting the network's change dynamically, is a computing tool that can solve some question such as complex, dynamic and asynchronous problems.

Along with the development of bionics and agent, ant colony algorithm and agent is applied to network's routing gradually. The first ACO routing algorithm was designed for wired networks like as AntNet, which is a proactive routing protocol, mainly using ants to discover and maintain route, but it doesn't adapt the networks that constantly change, so doesn't apply to Ad Hoc networks.

The ACO routing algorithms were designed for MANET such as Ant-AODV and AntHocNet all use mobile agents like ants to carry on big scope to scan the networks, make use of ant agents to collect the information about routing. Among them, the Ant-AODV combines ACO proactive characteristics with AODV reactive characteristics, to improve the performance of routing search delay and to adapt the constantly change of network's topology; AntHocNet is a hybrid algorithm, which combines reactive path setup with proactive path probing, maintenance and improvement. Other ACO routing of MANET like GPS/AL, ANSI, PERA, ARAMA, MANSI, Termite etc, they all have their own applied area[1].

In addition to laying pheromone on the path an ant moves, to make ACO metaheuristic suitable for MANET QoS routing, the ant additionally senses and collects some of the environment related parameters like number of nodes the ants have visited, the delay taken to reach destination and to come back to source and the available bandwidth of each link it has traveled. When the ant comes back to source, all details collected by that ant will be used to find whether the path taken by it will be suitable for real time data transmission or not. If suitable then the path will be selected for data transmission after doing soft resource reservation on all intermediate nodes along the path[2].

\section{Chaos ant Pheromone Model}

MANET routing is a difficult problem because network characteristics such as traffic load and network topology may vary stochastically and in a time varying nature. As we can see, the distributed nature of network routing is well matched by the multi agent nature of ACO algorithms. ACO adopts the foraging behavior of real ants. Initially when there is no neighborhood relationship between two nodes, the pheromone substance on that link will be zero. As soon as neighborhood relationship is established between two nodes, $i$ and $j$, an initial pheromone amount 0.1 is deposited on that link as

$$
\tau_{i j}=0.1
$$

To avoid searching blindly before reducing the searching space of variable optimized in chaos optimization algorithm, a Chaos algorithm is introduced. Chaos is a kind of random motion state, which is obtained by a certain equation. Logistic mapping is a typical chaos system, and iterative method is $z_{i+1}=\mu z_{i}\left(1-z_{i}\right), i=0,1, \cdots ; \quad \mu \in(2,4]$ While $\mu=4,0 \leq z_{o} \leq 1$, Logistic is a chaos state. For each Route_Reply_Ant received by an intermediate node ${ }^{i}$ from node ${ }^{\bar{j}}$, the link $i_{\text {to }} j$ is positively reinforced as follows [5]. $\tau_{i j}=\tau_{i j}+\Delta \tau_{i j}+q Z_{i j}$

where $\Delta \tau_{i j}=0.05, Z_{i j}$ is chaos variant, and $q$ is coefficient.

There is also an implicit negative reinforcement for the pheromone values. Within every finite time interval $\mathrm{T}$ delay, if there is no data toward a neighbor node, its corresponding pheromone value decays by a factor $\rho$ as follows.

$$
\tau_{i j}= \begin{cases}(1-\rho) \tau_{i j} & \text { if }(1-\rho) \tau_{i j} \succ 0.1 \\ 1 & \text { if }(1-\rho) \tau_{i j} \geq 1 \\ 0.1 & \text { otherwise }\end{cases}
$$

When node ${ }^{i}$ losses its connectivity to its neighbor ${ }^{j}$, the pheromone on the link $i_{\text {to }}{ }^{j}$ will be set to 0 .

\section{Path Preference Probability Model}

Path preference probability is calculated in both intermediate nodes as well as source nodes upon receipt of Route_Reply_Ant. Suppose current node $i$ receives a Route_Reply_Ant from node $j$ for destination $d$ then, the path preference probability is calculated as follows [2].

$$
P_{i j d}=\frac{\left[\tau_{i j}\right]^{\alpha}\left[D_{i j d}\right]^{\beta}\left[\eta_{i j d}\right]^{\gamma}\left[B_{i j d}\right]^{\delta}}{\sum_{l \in N_{i}}\left[\tau_{i l}\right]^{\alpha}\left[D_{i l d}\right]^{\beta}\left[\eta_{i l d}\right]^{\gamma}\left[B_{i l d}\right]^{\delta}}
$$

Where $\alpha, \beta, \gamma$ and $\delta(\geq 0)$ are tunable parameters that control the relative weight of pheromone trail $\tau_{i j}$, Delay $D_{i j d}$ and hop count considered as heuristic value $\eta_{i j d}$, 
bandwidth $B_{i j d}$ respectively, also, $N_{i}$ is the set of neighbors of $i$ and $l$ is neighbor node of $i$ through which a route is available to destination $d$.

The relative metrics are calculated (using (13), (14) and (15)) as follows when next hop on the path from $i$ to $d$ is $j$.

$$
\begin{gathered}
D_{i j d}=\frac{1}{\operatorname{delay}(\operatorname{path}(i, d))} \\
\eta_{i j d}=\frac{1}{\operatorname{hopcount}(\operatorname{path}(i, d))} \\
B_{i j d}=\frac{1}{\operatorname{bandwidth}(\operatorname{path}(i, d))}
\end{gathered}
$$

After finding the path preference probability through various neighbors, the source as well as intermediate nodes now have multiple paths to the destination. The path which has higher path preference probability will be selected for data transmission.

\section{EXPERIMENT AND PERFORMANCE ANALYSIS}

The experiment results are shown in figure 1 and figure 2. The performance of the proposed was compared with another on demand state of the art routing protocol AODV for the same network and load characteristics. 100 nodes using the typical Random Waypoint Model moves within a $2500 \mathrm{~m} * 1500 \mathrm{~m}$ area with Mobility Speed: $1-20 \mathrm{~m} / \mathrm{s}$ respectively. The mechanism of IEEE802.11 DCF is used by MAC layer, thus channel capacity is $2.5 \mathrm{Mbps}$. There are 40 CBR sources each send 5 data packet per second. The size of data payload is 512 bytes per packet. The other parameters are set as follow: Two way ground, radio range of single node: 400 Meters, and the simulation lasts 900 seconds of simulation time to collect the statistics of end-to-end delay, packet successfully delivery ratio.

Fig. 1 shows the effect of pause time on end-to-end delay of AODV and proposed protocols. At higher pause times, the nodes stay longtime in each waypoint and hence path breaks will be lesser. So with higher pause times, both algorithms provide almost same and low end-to-end delays.

Fig. 2 shows the effect of packets received for both the protocols in terms of mobility. In both cases higher the node mobility, lesser is the packet delivery. Because in such cases there will be frequent path breaks and hence frequently route establishment phase will be invoked in case of AODV.

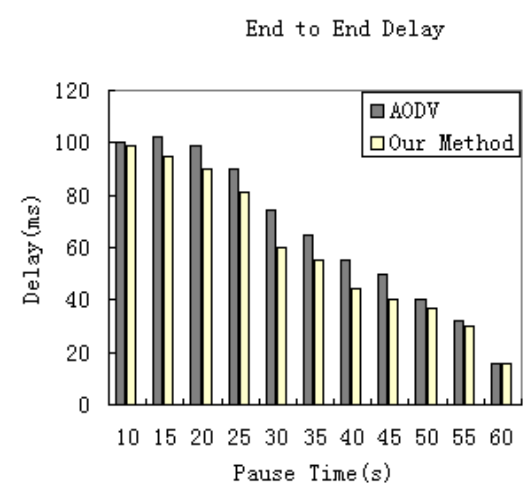

Figure 1. Effect of Pause Time on end-to-end delay

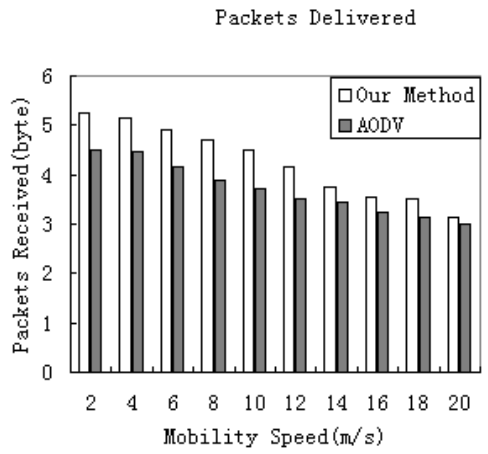

Figure 2. Effect of mobility on packets received

\section{CONCLUSION}

In this paper, we have proposed a novel QoS constraintsbased multicast routing algorithm using CACO. The approach is based on swarm intelligence and especially on the chaos ant colony optimization meta-heuristic. We compared the performance of the proposed protocol with that of the AODV routing protocol in terms of the pause time on end-to-end delay, the mobility on packet received,etc. Our simulation results show that the proposed protocol perform well than the conventional AODV under high mobility because of alternate route maintenance scheme.

\section{ACKNOWLEDGMENT}

This work is sponsored by the .National Natural Science Foundation of China (Grant No.60873075), the China 863 National High Technology R\&D Program (Grant No. 2009AA01Z422)

\section{REFERENCES}

[1] Wan-Jun Yu, Guo-Ming Zuo, Qianq-Qian Li. Ant colony optimization for routing in mobile ad hoc networks. 2008 International Conference on Machine Learning and Cybernetics, 2008, pp.1147-1151

[2] P.Deepalakshmi, Dr.S.Radhakrishnan. QoS Routing Algorithm For Mobile Ad Hoc Networks. International Journal of Recent Trends in Engineering, 2009, 5(1): 459-462. 
[3] Yingzhuang Liu, Hong Zhang, Qiang Ni. et al. An Effective AntColony Based Routing Algorithm for Mobile Ad-hoc Network. The 4th IEEE International Conference on Circuits and Systems for Communications, 2008, pp.100-103

[4] Kewen Li, Jing Tian. A QoS Mobile Multicast Routing Algorithm Based Ant Colony Algorithm. 2008 IEEE Pacific-Asia Workshop on Computational Intelligence and Industrial Application, 2008, pp. 8892

[5] M Dorigo, M Birattari ,T Stutzle, Ant colony optimization: Artificial ants as a computational intelligence technique, IEEE Transaction on Computational Intelligence, 2006, 11(4): 28-39
[6] Ran Min, Suixiang Gao, Xu Bao. A Multi-constrained QoS routing algorithm based on the Ant Colony Algorithm.Computer engineering and application, 2005.7:142 - 144

[7] D. Kim, "A New Mobile Environment: Mobile Ad Hoc Networks (MANET),” IEEE Vehic. Tech. Soc. News, Aug. 2003, pp. 29-35.

[8] J. N. Al-Karaki and A. E. Kamal, "Quality of Service Routing in Mobile Ad Hoc Networks: Current and Future Trends," Mobile Computing Handbook, I. Mahgoub and M. IIays, Eds., CRC Publishers, 2004.

[9] Zheng Shao-ren;Wang Hai-tao,Zhao Zhi-feng,etc. Ad Hoc Network Technology [M]. Peiking:POSTS \& TELCOM PRESS, 2005,2-3 (in Chinese) 